\title{
EFFECTS OF SAMPLE PREPARATION IN ANALYSIS: IMAGING
}

\author{
C. Barry Carter, Shelley R. Gilliss, and Christopher R. Perrey
}

Dept. of Chemical Engineering and Materials Science, University of Minnesota

421 Washington Ave. S.E., Minneapolis MN 55455 USA

The Achilles heel of transmission electron microscopy has been, and still is, the requirements of sample preparation (see [1]). Some samples are already sufficiently thin and do not require further thinning, for example nanoparticles and nanotubes, but often numerous steps must be used to take a specimen from the bulk to a state of electron transparency. This paper will examine some of the 'traditional' artifacts and then consider artifacts which may arise in newly developed thinning techniques such as the use of FIB systems. The almost classical examples of artifacts due to thin foils include the following:

- Dislocations which are parallel or inclined to the sample surface

- Amorphization of interfaces and surface regions by ion milling

- Grooving at interfaces by chemical or ion thinning

- Implantation effects or the preferential removal of a component due to ion milling

Each of these effects can influence both the chemistry and the structure of the material; the chemical analysis will be discussed in a companion paper [2]. It is important also to distinguish artifacts which arise during observation in the TEM although some of these may also be influenced by the specimen preparation. Figure 1 shows a HREM image (JEOL 3000F) of a nanocrystal of silicon contained in an amorphous matrix of silicon exhibiting icosahedral symmetry. This morphology is not a result of the fabrication process used to make this film, but a result of the electron beam interacting with the sample. Careful analysis and repeated observations proved that this film did contain nanocrystallites of $\mathrm{Si}$, but these were only of the order of 2-3 $\mathrm{nm}$ in size [3].

Although the rapid rise in the use of the focused-ion beam (FIB) tool has eased some of the burden of specimen preparation, this relatively new technique shares many disadvantages with that of traditional TEM specimen preparation. The interpretation of TEM images requires that the microscopist be aware of the possibility of artifacts in images which may have been caused by specimen preparation. Often, imaging alone will not confirm or even illustrate artifacts caused by sample preparation; a combination of imaging and chemical analysis must be performed to ensure the contrast and morphology observed are truly inherent to the specimen. These sometimes elusive features in images can mislead and confound researchers.

Figure 2, shows a dark-field (DF) TEM image (Philips CM30) of a FIB-prepared specimen of silica glass which has dewet the (110) surface of rutile. Contrast seen in the silica glass layer could suggest the presence of precipitated titanium oxide in the silica glass. However, electron energy-loss (EELS) and X-ray energy dispersive spectroscopy (XEDS) confirm that this contrast is actually a result of damage created during the Pt-deposition process (deposited to protect the specimen during FIBing). Unfortunately, the Pt deposition itself can lead to amorphization and implantation of Pt into a specimen. The FIB is an ideal tool for preparing specimens of grain boundaries which contain amorphous films. Conventional ion milling and chemical etching may produce different removal rates of the matrix and boundary materials. Figure 3 is an annular dark-field (ADF) STEM image (Philips CM200 FEG) of an intergranular layer in an alumina bicrystal. The origin of this specimen would assert that the intergranular film is anorthite glass $\left(\mathrm{CaSi}_{2} \mathrm{Al}_{2} \mathrm{O}_{8}\right)$ which was used in the processing of the bicrystal. This amorphous film appears that it has dewet the grain boundary during the cooling process. Following the trajectory noted in Figure 3 a XEDS line profile was performed. Figure 4 is a plot of the Ga and Ca XEDS signals along this scan. It is clear from the lack of Ca and relatively large $\mathrm{Ga}$ signal in the boundary that the glass layer has been destroyed by the milling process. 
With care, specimens can be successfully prepared by FIB [3-5]. It is notable that traditional preparation methods such as mechanical or chemical thinning and Ar ion milling could reasonably lead to similar results for both of the above examples.

\section{References}

1. D.B. Williams, and C.B. Carter, Transmission Electron Microscopy: A Textbook for Materials Science. Plenum, New York, 1996.

2. D.B. Williams and M. Watanabe, Microsc. Microanal., 2003. 9(suppl. 2) in press.

3. C.R. Perry et al., 2003. 9(suppl. 2) in press.

4. N. Ravishankar et al., Microsc. Microanal., 2002. 8(4): 257.

5. S.R. Gilliss et al., Microsc. Microanal, 2002. 8(suppl. 2) 562.

6. C.R. Perrey et al., Microsc. Microanal. 2002. 8(suppl. 2) 1144.

7. This work is supported by DOE grant DE-FG02-01ER45883 and NSF grant DMI-0103169. The authors thank Jim Bentley, John Hutchison and Markus Lentzen for discussions.

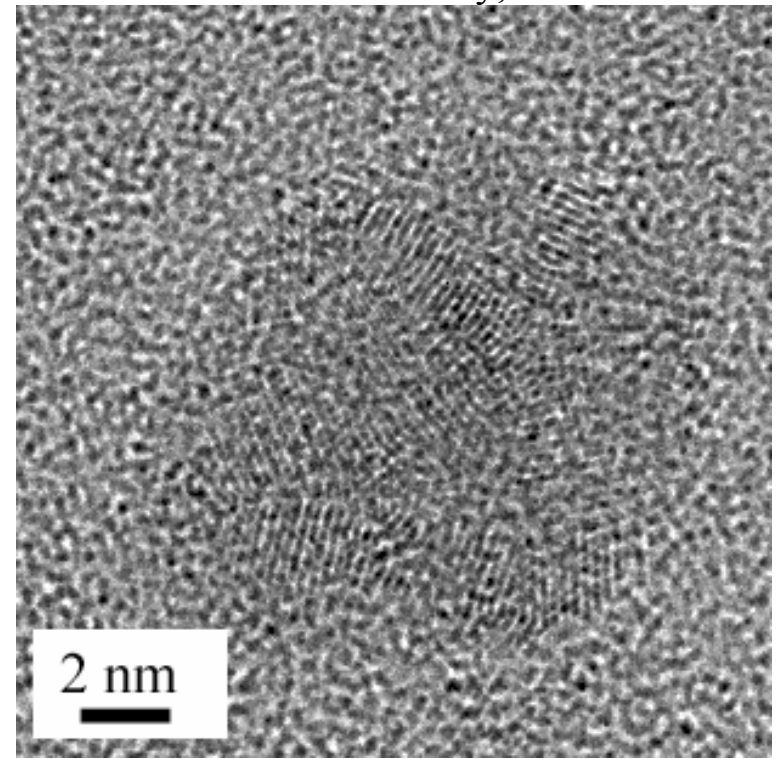

FIG. 1 HREM image of a $\mathrm{Si}$ nanocluster in an amorphous $\mathrm{Si}$ matrix. The icosahedral nanocluster is formed by beam interactions.

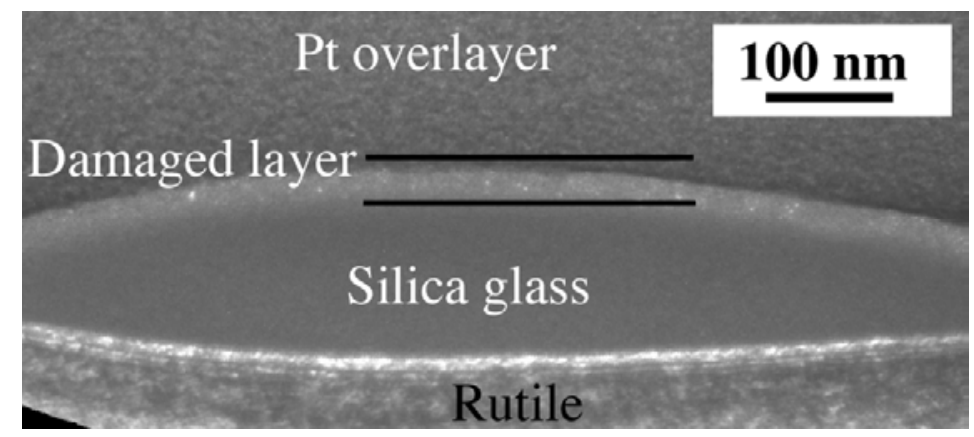

FIG. 2 DF TEM image of a dewet silica droplet on the surface of (110) rutile. The TEM specimen exhibits a damaged region with apparent crystallites. This originates from Pt deposition.

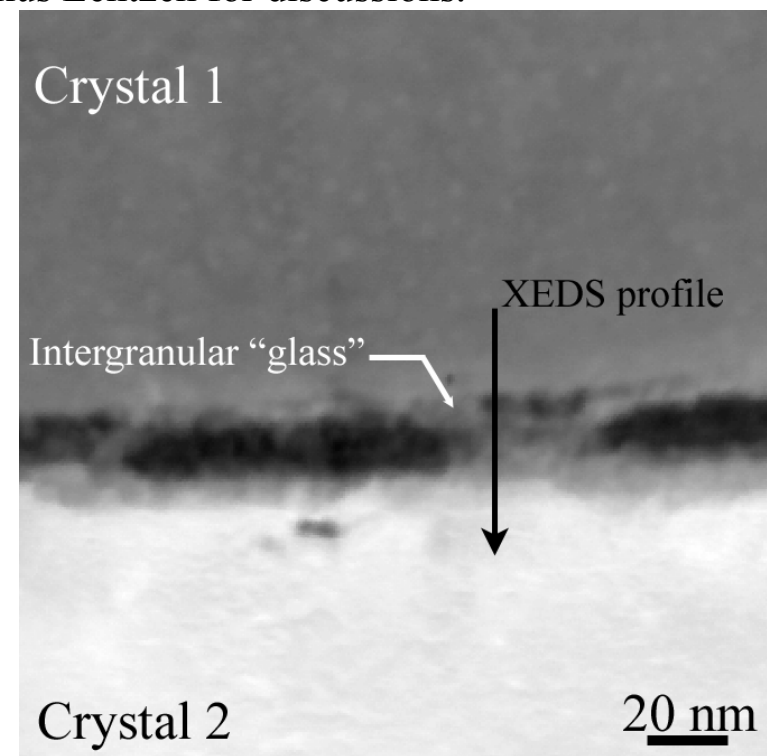

FIG. 3 ADF STEM image of an alumina bicrystal that appears to have an intergranular glass layer. The trace for a spectral profile is shown. CM200 FEG.

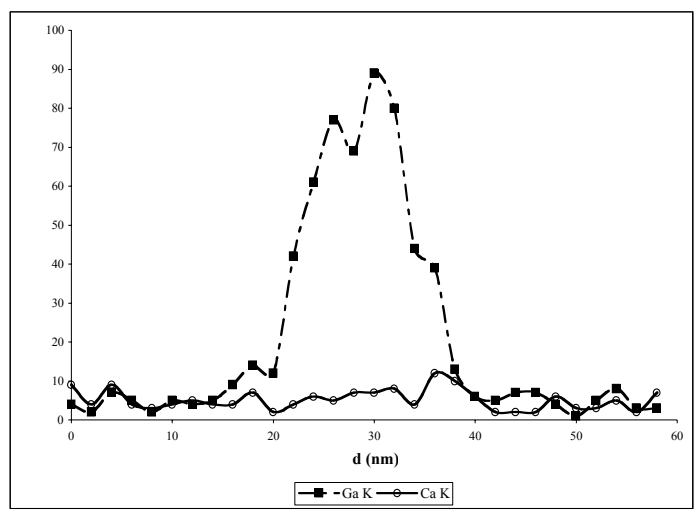

FIG. 4 XEDS spectra (trace shown in Fig. 3). Concentrations of $\mathrm{Ga}$ and $\mathrm{Ca}$ in the "glass" result from $\mathrm{Ga}$ implantation and $\mathrm{Ca}$ removal in the intergranular layer. CM200 FEG 